\title{
Automatic Guided Waves Data Transmission System Using an Oil Industry Multiwire Cable
}

\author{
Gianpiero Trane $^{1}$, Rito Mijarez ${ }^{2, *(1)}$ and Jesús Arturo Pérez-Díaz ${ }^{1}$ \\ 1 Tecnologico de Monterrey, Escuela de Ingeniería y Ciencias, Colonia Real del Puente C. P. Xochitepec 62790, \\ Morelos, Mexico; a01125467@itesm.mx (G.T.); jesus.arturo.perez@tec.mx (J.A.P.-D.) \\ 2 Instituto Nacional de Electricidad y Energías limpias, Gerencia de Control, Electrónica y Comunicaciones, \\ Calle Reforma 113, Col. Palmira C.P. Cuernavaca 62490, Morelos, Mexico \\ * Correspondence: rmijarez@ineel.mx; Tel.: +52-777-362-3811
}

Received: 9 January 2020; Accepted: 30 January 2020; Published: 6 February 2020

\begin{abstract}
Alternative wireless data communication systems are a necessity in industries that operate in harsh environments such as the oil and gas industry. Ultrasonic guided wave propagation through solid metallic structures, such as metal barriers, rods, and multiwire cables, have been proposed for data transmission purposes. In this context, multiwire cables have been explored as a communication media for the transmission of encoded ultrasonic guided waves. This work presents the proprietary hardware design and implementation of an automatic data transmission system based on the propagation of ultrasonic guided waves using as communication channels a high-temperature and corrosion-resistant oil industry multiwire cable. A dedicated communication protocol has been implemented at physical and data link layers, which involved pulse position modulation (PPM), digital signal processing (DSP), and an integrity validation byte. The data transmission system was composed of an ultrasonic guided waves PPM encoded data transmitter, a $1 \mathrm{~K} 22 \mathrm{MP}-35 \mathrm{~N}$ multiwire cable, a hardware preamplifier, a data acquisition module, a real-time (RT) DSP LabVIEW (National Instruments, Austin, TX) based demodulator, and a human-machine interface (HMI) running on a personal computer. To evaluate the communication system, the transmitter generated $60 \mathrm{kHz}$ PPM energy packets containing three different bytes and their corresponding integrity validation bytes. Experimental tests were conducted in the laboratory using 1 and $10 \mathrm{~m}$ length cables. Although a dispersive solid elastic media was used as a communication channel, results showed that digital data transmission rates, up to $470 \mathrm{bps}$, were effectively validated.
\end{abstract}

Keywords: piezoceramic transducers; guided waves-based communication; pulse position modulation; digital signal processing; multiwire cables

\section{Introduction}

Harsh environments characterize cased hole production engineering structures in the hydrocarbons sector, for example, downhole oil reservoirs either onshore or offshore could be immersed underwater or in corrosive liquids and high-temperature high-pressure boreholes' surroundings [1]. Downhole data acquisition from a producing well is intended primarily to measure the performance of a well in production, better understand the dynamic behavior, plan remedial work if it is required, and optimize the long-term production. The production measurements are flow, density, temperature, and pressure. Hence, no hydrocarbon can be produced without the intervention of these measurements $[2,3]$. The acquisition of these bottom hole geophysical parameters using digital data transmissions under these aggressive conditions has become difficult, and the use of cables dedicated exclusively to communication media and conventional radio wave wireless communications has proven to be impractical $[4,5]$. The instrument that is descended into a wellbore to measure geophysical parameters 
must be able to endure the extreme hole conditions that could be encountered. The instrument is connected to a surface station, generally controlled by a computer, via a cable. Data acquisition should be performed with minimum disturbance to the normal operating production procedures. Detailed records, also termed well $\mathrm{log}$, are surface read-out measurements that are achieved either in real time using electrical signals via power line communications and a multiwire cable as communication channel, or the data can be stored downhole in a bottom hole instrument operated by batteries and reviewed when it is retrieved later. In the former approach, downhole environmental factors, mainly high temperature, increase the electrical resistance in the cables and modify the behavior of the passive electrical components, for example, imposing important deviations in central frequencies of front-end filters, thereby compromising the propagation of electrical signals for data transmissions [6-8]. The advantages of the latter approach include lower operating costs and a fixed operations schedule. The main shortcoming is the possibility of important operating losses if the bottom hole recording instrument malfunctions. However, real-time downhole measurements that employ multiwire cables are recognized, in the petroleum industry, as more important for making prompt decisions [3].

Guided waves have been a relevant tool for non-destructive testing (NDT) and structural health monitoring (SHM) technologies due to their wide screening of the acoustic field and their ability to propagate long distances in large structures [9-11]. SHM of multiwire cables using ultrasonic guided waves has been investigated numerically and experimentally with a particular focus on damage detection [12-19]. Moreover, the reuse of metallic industry infrastructure has been proposed by several research groups as a communication medium for digital transmission systems using field-proven guided waves that are excited and received either by piezoelectric or electromagnetic transducers. The methods employed for modulation include frequency-shift keying (FSK), amplitude-shift keying (ASK), chirp on-off keying (Chirp-OOK), pulse position modulation (PPM), orthogonal frequency-division multiplexing (OFDM) and code-division multiple access (CDMA), among others. Some researchers have used metal pipes [20-27], thick metal barriers [28-36], and plates [37-42] as communication channel.

Recently, the use of cables as a communication medium was proposed by Mateo et al. [43,44]. They used a single copper core PVC insulated electrical cable type H07V-U and two piezo ceramic discs, one as a transmitter and the other one as a receiver. The transmitter was excited via a train of chirp signals in the range of 1 to $12 \mathrm{MHz}$ and a signal averaging process was performed in the received signals. They achieved short-range communication of $0.25 \mathrm{~m}$ using a PVC insulated cable and $0.50 \mathrm{~m}$ by removing its dielectric PVC covers. Copper stranded cable 12 rating American wire gauge (12 AWG) has been used as a communication medium in a previous study [45,46]. In this approach, a digital data transmission system was developed that performed pulse position modulation (PPM) in the range of $60 \mathrm{kHz}$, to transmit eight bits of encoded information over a 12 AWG multiwire cable that was $1 \mathrm{~m}$ and $4 \mathrm{~m}$ in length. The aim was to achieve digital transmission of guided waves on mono-conductor multiwire cables commonly used in producing wells. The researchers also carried out preliminarily work in $1 \mathrm{~m}$ corrosion-resistant four conductors $4 \mathrm{H} 18$ multiwire cable, in which data rates in the order of 500 bits per second were accomplished by employing tone pulses of $60 \mathrm{kHz}$ and PPM [47].

In this study, we proposed a downhole instrument that is able to periodically transmit ultrasonic guided waves signals via a multiwire cable indicating that the instrument is operating correctly and sending complementary information related to the recorded bottom hole parameters. Hence, high-pressure and high-temperature corrosion-resistant mono-conductor multiwire cables $1 \mathrm{~K} 22$ MP-35N were employed. The periodicity of transmitted data, in this application domain, was either once every hour at the beginning of its operation or once per day when it reached a stable state at the bottom hole, depending on the operator requirements. Thereby, there was no need for stringent high data rates, and several transmitted bytes of information could suffice. A progressive approach was taken for the use of PPM and guided waves on this intricate mono-conductor multiwire cable. First, we achieved results using a low-cost electrical copper stranded cable 12 rating American wire gauge (12 AWG); secondly, we moved the investigation to a corrosion-resistant four-conductor cable $4 \mathrm{H}-18$ and guided waves; and finally, we used the intended mono-conductor $1 \mathrm{k} 22 \mathrm{MP}-35 \mathrm{~N}$ cable. The system 
consisted of an ultrasonic guided waves PPM encoded data transmitter, a hardware preamplifier, a data acquisition module, a real-time (RT) DSP LabVIEW based demodulator, purchased from National Instrument (NI) Mexico, and a human-machine interface (HMI) running on a personal computer. A dedicated communication protocol was implemented at physical and data link layers, which involved PPM, digital signal processing, and an integrity validation byte. To evaluate the communication system, the transmitter generated $60 \mathrm{kHz}$ PPM energy packets containing three different bytes and their corresponding integrity validation bytes. Experimental tests were conducted in the laboratory using 1 and $10 \mathrm{~m}$ length cables. Automatic digital data transmission rates up to $470 \mathrm{bps}$ were successfully achieved. In this paper, the panorama of research and detailed significant characteristics of the novel automatic data transmission guided waves system is described. The proprietary hardware design of the original battery-operated stand-alone electronics instrumentation for the guided waves transmitter is presented, including its schematic diagram; and on the receiver side, the performed signal processing algorithms are detailed.

\section{Guided Wave Propagation in Multiwire Cables}

Multiwire cables are more complicated mechanical structures than individual solid cylindrical rods. The complex interaction between multiple wires due to the geometry of its cross-section is its most important feature. 1K22 MP-35N cables are multiwire structures made of an inner copper construction, a plastic insulation layer, and a steel armor that can withstand high-temperature, high-pressure, and corrosive environments. Figure 1a,b shows an actual 1K22 MP-35N cable and CAD model, respectively, and Figure $1 \mathrm{c}$ illustrates the composition of the wires. The inner construction is comprised of seven individual nickel-coated copper 18 AWG wires of $0.404 \mathrm{~mm}$ diameter. Voids in the copper strands are filled with a water-blocking agent to reduce water and gas migration. The copper construction is covered in a perfluoroalkoxy (PFA) plastic-type isolation layer that is $0.713 \mathrm{~mm}$ in thickness. The armor is made up of two types. The former is an inner armor of 15 wires that are $0.617 \mathrm{~mm}$ in diameter, and the latter is an outer armor of 15 wires that are $0.909 \mathrm{~mm}$ in diameter. Both armors are nickel-cobalt corrosion-resistant stainless-steel wires, which are coated with an anti-corrosion compound for protection during shipping and storing. A special pressure seal agent, SuperSeal (Canusa-CPS, Toronto, ON, Canada), is applied between the armor layers [48].

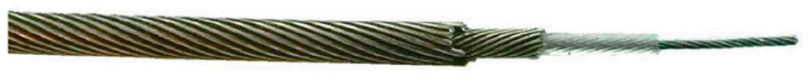

(a)

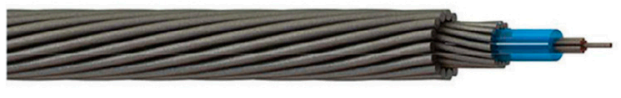

(b)

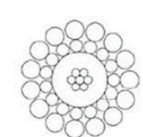

(c)

Figure 1. (a) Actual $1 \mathrm{~K} 22 \mathrm{MP}-35 \mathrm{~N}$ corrosion-resistant cable; (b) $1 \mathrm{~K} 22 \mathrm{MP}-35 \mathrm{~N}$ CAD model; and (c) $1 \mathrm{~K} 22 \mathrm{MP}-35 \mathrm{~N}$ construction.

To the best of our knowledge, an analytical solution that describes the wave propagation in these complex multiwire cables does not exist. A practical approach to understand guided wave propagation in multiwire cables is based on the study of wave propagation behavior of an individual wire using the analytical Pochhammer-Chree frequency equation which has been discussed in detail by Graff [49] and more recently by Rose and Nagy [50]. The solutions of the elastic equation of motion yield an infinite number of guided waves modes, which are categorized by their particle displacement, and can well propagate through the structure. These modes in a cylindrical waveguide correspond to the longitudinal waves $\mathrm{L}(0, \mathrm{n})$ with radial and axial vibration displacement, torsional waves $\mathrm{T}(0, \mathrm{n})$ with circumferential vibration displacement, and flexural waves $\mathrm{F}(\mathrm{M}, \mathrm{n})$ with radial, axial, and circumferential vibration displacement, where $M$ refers to the circumferential order and $n$ 
represents the nth root of the characteristic equation. All possible propagating wave modes in a cable at different frequencies can be predicted by guided waves dispersion curves, which can be obtained by solving the Pochhammer-Chree frequency equation, via the commercial software Disperse [50].

The approach taken by researchers of previous studies consisted of modeling two different multiwire cables, an AWG 12 cable and a $4 \mathrm{H} 18$ cable, as a concentric rod composed of different materials $[45,47]$. The model considered that the wavelengths of the propagating guided waves employed was significantly larger than the small gaps between the strands. Cheng et al. also modeled, in this manner, an aluminum conductor steel reinforced (ACSR) multiwire cable [18]. In this work, a multiwire $1 \mathrm{~K} 22 \mathrm{MP}-35 \mathrm{~N}$ cable was modeled as a rod made of copper that was $1.212 \mathrm{~mm}$ in diameter, surrounded by an isolation layer of PFA that was $0.713 \mathrm{~mm}$ in thickness, and coated with steel armor that was $1.526 \mathrm{~mm}$ in thickness, as depicted in Figure 2a. The group velocity dispersion curves for this concentric rod calculated by the software Disperse are plotted in Figure $2 \mathrm{~b}$.

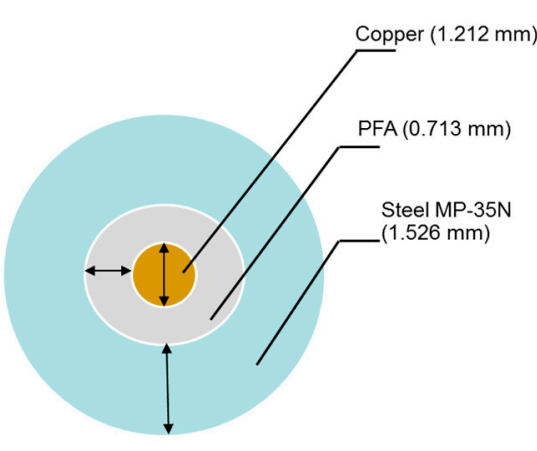

(a)

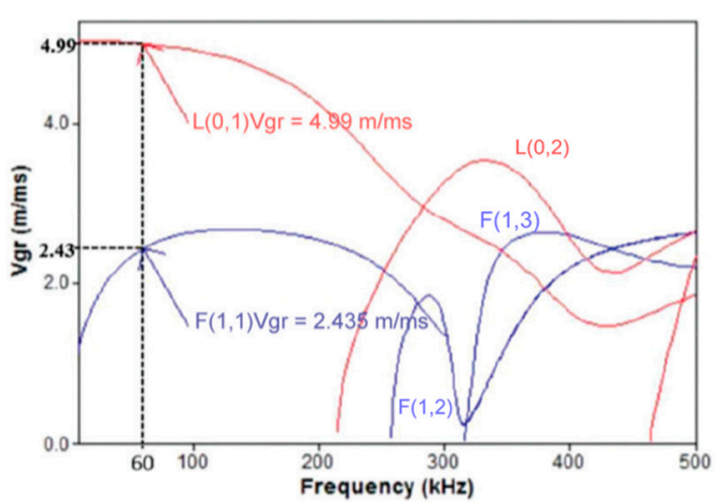

(b)

Figure 2. (a) Cable cross-section considerations to solve the Pochhamer-Chree frequency equation; (b) dispersion curves of a $1.212 \mathrm{~mm}$ diameter copper rod surrounded by a perfluoroalkoxy (PFA) of $0.713 \mathrm{~mm}$ thickness and steel armor of $1.526 \mathrm{~mm}$ thickness.

Frequency and Signal Selection for Guided Waves Excitation

The propagation of guided waves has been researched in three regimens, short $(<<1 \mathrm{~m})$, medium (up to about $5 \mathrm{~m}$ ), and long range (above $100 \mathrm{~m}$ ). In long-range structures, frequencies below $100 \mathrm{kHz}$ are generally required, as validated by Cawley et al. [51]. However, when working with frequencies lower than $100 \mathrm{kHz}$, the audio bandwidth that can lead to interference and a poor SNR. Studies have been carried involving the propagation of 20 and $40 \mathrm{kHz}$ guided waves energy packages in tubes [21,23] and $60 \mathrm{kHz}$ guided waves pulses in electrical 12 AWG cables [45,46], and preliminarily in $4 \mathrm{H} 18$ multiwire corrosion-resistant cables [47]. As shown in Figure 2b, for a concentric rod copper-PFA-steel, below $200 \mathrm{kHz}$, the assortment of the modes presents less difficult since there are only two possible excited modes, $\mathrm{L}(0,1)$ and $\mathrm{F}(1,1)$. Hence, the frequency selection depends on the transducer device and its frequency spectrum. In this work, piezo ceramic discs with normal beam loading and reception have been employed, thereby it is anticipated that longitudinal modes and flexural modes are excited depending on the pressure distributions exerted on the surface of the cable.

NDT and SHM long-range applications commonly use narrowband signals to provide suitable signal strength and prevent dispersion. Single-frequency sinusoidal waves of between five and ten cycles, modulated by a Hanning or a Gaussian window, are often employed [51,52]. The use of square pulses produces an increase in the energy of the transmitted signals enhancing the SNR, as has been validated by Pollakowski et al. [53]. Square pulses contain higher harmonics that can produce different speeds than the carrier frequency, which can lead to dispersion in an elastic media and multiple mode excitations. However, piezoelectric (PZT) transducers possess a frequency response similar to band-pass filters, with a bandwidth that depends on the transducer design. Hence, only the carrier frequency plus the frequency components that enter the transducer bandwidth can go through. In this 
application, the use of square wave pulses to excite PZT elements that possess a narrow bandwidth ( $3 \mathrm{~Hz}$ ) yields an additional gain to the transmitted guided waves signal. The electronics required for the generation of square wave pulses presents two advantages that simplify its design. First, the square waves can be achieved readily using high-speed and highly integrated digital circuits such as microcontrollers, avoiding the employment of digital-to-analogue converters and low pass filters. Secondly, the power amplifiers that are normally used to increase the voltage signals applied to PZT elements, can be substituted for simple signal boosters, for example, $\mathrm{H}$-bridge driver circuits. Considering these aspects and the experience of researchers in previous works on multiwire cables, square pulses of $60 \mathrm{kHz}$ were used for powering piezo ceramic PZT-5H elements.

\section{Pulse Position Modulation Communication}

PPM is an efficient modulation technique that has been used by several research groups to transmit digital information via guided waves $[23,24,27,47]$. In this application, PPM has been applied in the decoding and encoding process to attain data transmission using guided waves through multiwire structures. PPM is an attractive modulation due to its reduced sensitivity to multipath propagation; its simplicity for power-efficient channels, theoretically, PPM systems are effective when the signals are power limited rather than band limited, as a result of the transmission of $\mathrm{k}$ bits for the same average power [54]; and the instrumentation required for its implementation is simple and lightweight. PPM is a modulation scheme in which the message information is modulated in the time slot between pulses, $M$, in a sequence of signal pulses. In PPM, a symbol can be represented by

$$
k=\log _{2}(M)
$$

where $k$ is the number of bits. A symbol contains a single pulse in the position indicated by the binary representation of the data codeword, thus, $k / M$ bits are transmitted per time slot [55]. In general, the PPM generation of signals, $S_{m}(t)$, is given by [56]:

$$
S_{m}(t)=\varphi\left(t-m T_{0}\right) ; m=\frac{(-1)^{b}}{2}
$$

where $\varphi(t)$ is a unit energy pulse that is similar to those found in the sampling theorem, $T_{0}$ is the time slot for each digital bit representation, and $\mathrm{m}$ stands for the temporal displacement of an acoustic pulse quantified for half of the time slot $T_{0}$. The coefficient $b$ represents the digital value of each bit transmitted within its time slot $T_{0}$. The direction of the temporal displacement $\mathrm{m}$, from $-T_{0}$ to $T_{0}$, is given by the digital value of the transmitted bit. For displacements with an increment of time, $+m$, the modulation corresponds to the logical level zero (bit 0 ) and for displacements with a decrement of time, $-m$, the modulation represents the logical level one (bit 1 ). As an example, a sequence of pulses that are not modulated is depicted in Figure 3a, where each pulse is exactly in the center of a time slot. Figure $3 \mathrm{~b}$ shows a sequence of pulses displaced $\pm \mathrm{m}$, according to the PPM in a 0110 sequence of bits. 
(a)

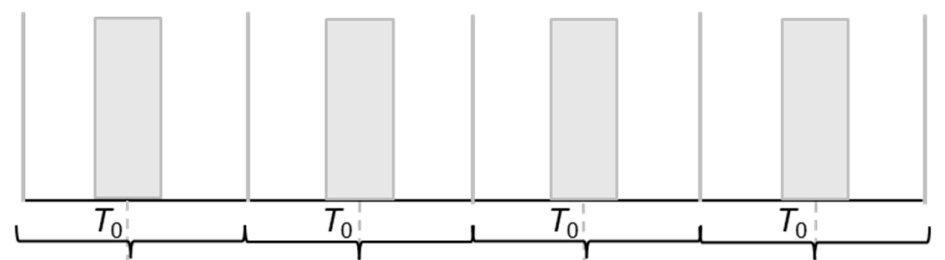

(b)

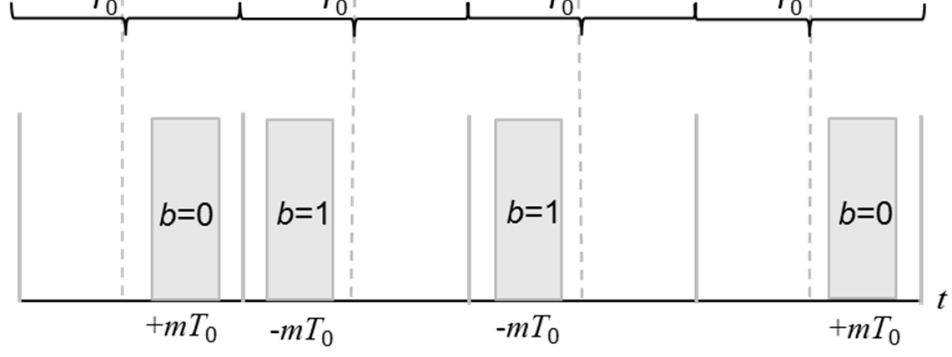

Figure 3. (a) Sequence of pulses without modulation and (b) sequence of pulse position modulation (PPM) pulses (0110).

\section{Automatic Guided Waves Data Transmission System}

The overall automatic guided waves data transmission system is illustrated in Figure 4. The system consists of a microcontroller-based piezo-ceramic transmitter as PPM generator, 1K22 MP-35N multiwire cable as communication channel, and an amplifier and a commercial data acquisition LabVIEW based modules connected to the HMI running in a computer as PPM receiver for automatic PPM demodulation.

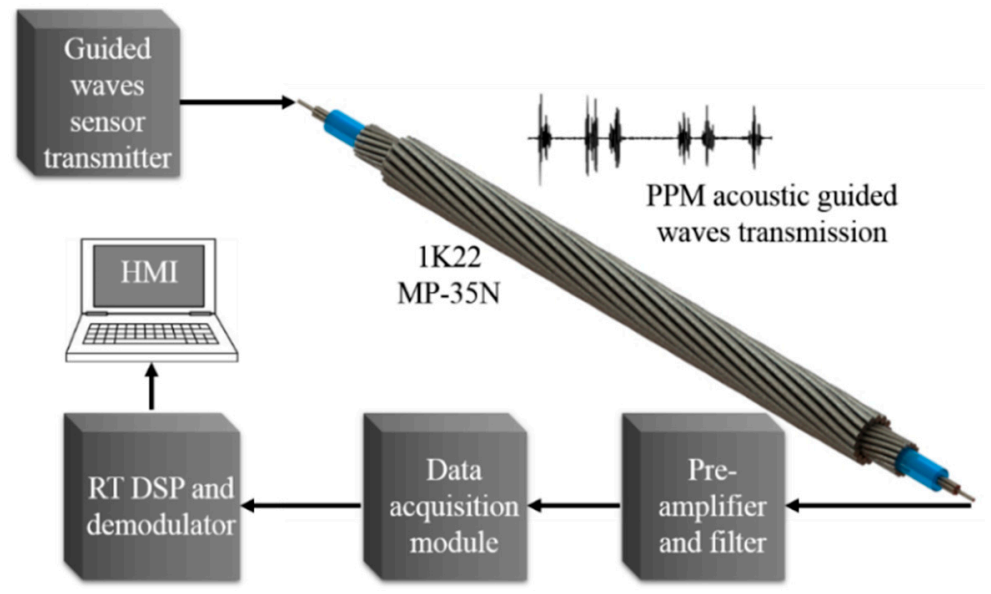

Figure 4. Automatic data transmission guided waves communication system block diagram.

\subsection{PPM Signal Transmitter}

The PPM generator, working as guided waves transmitter, is a stand-alone battery-operated electronic module composed of a microcontroller, a half-H driver, and a piezo ceramic PZT-5H disc, as is shown in Figure 5.

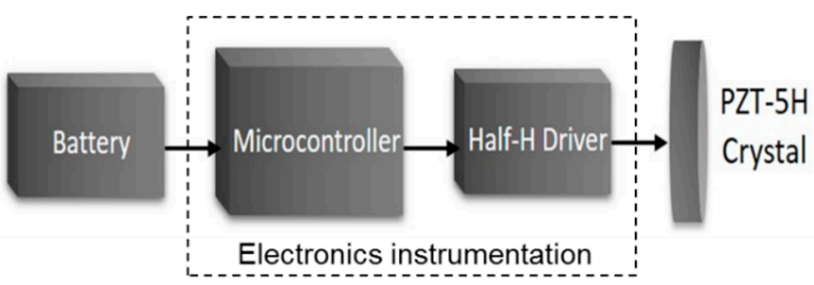

Figure 5. Block diagram of the PPM signal generator design used as a transmitter. 
The electronics instrumentation is a simple design that possesses relatively little weight. The electronics circuit is made of three integrated circuits, i.e., a microcontroller, a half-H driver, and a voltage regulator. Figure 6 depicts its schematic diagram, printed circuit board (PCB) design, and the actual PPM generator.

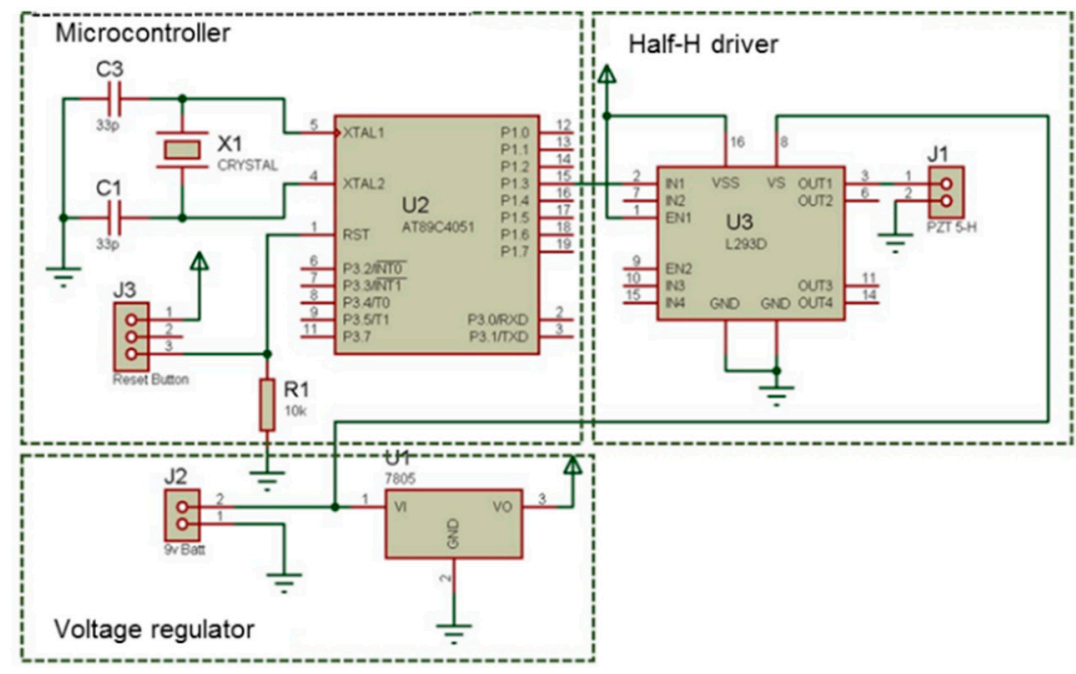

(a)

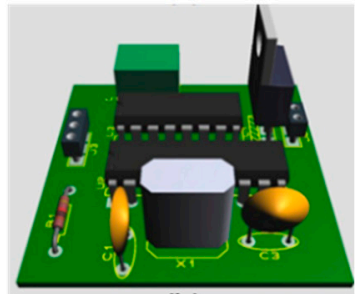

(b)

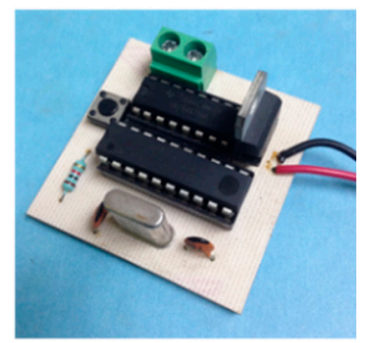

(c)

Figure 6. Electronics design and construction of the PPM generator. (a) Schematic diagram; (b) PCB design; and (c) actual PPM generator.

The components employed in the assembly of the PPM generator are a $9 \mathrm{~V}$ battery, the electronics instrumentation, and the PZT-5H crystal disc $38 \mathrm{~mm}$ in diameter and $5 \mathrm{~mm}$ in thickness, which were mounted in a cylindrical case made of a polymer called Nylamid (Nylene, Wayne, NJ, USA) with an inner diameter of 2 inches and an outer diameter of 3 inches. The case was fixed to a metallic support and base, as depicted in Figure 7.

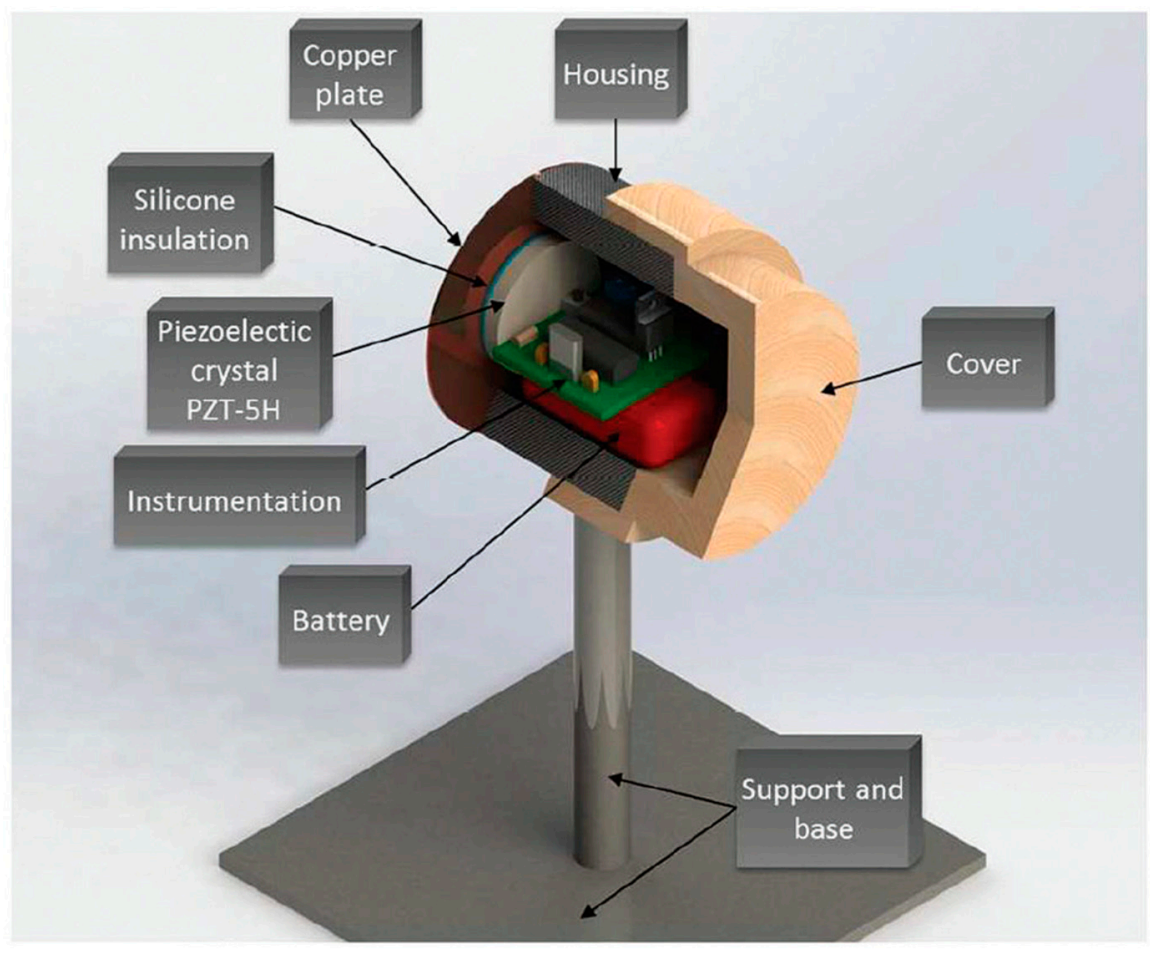

Figure 7. PPM generator components. 
The PZT-5H crystal connected to the electronic instrumentation was glued using silicone to an external copper plate 3 inches in diameter and $1 \mathrm{~mm}$ in thickness. One side of the copper plate was fixed to the case and the other was soldered to the copper cables of the $1 \mathrm{~K} 22 \mathrm{MP}-35 \mathrm{~N}$ cable. Details of the PPM generator construction have been previously described by [57].

The program that generates the PPM encoded words was programmed into the internal flash memory of the microcontroller in the instrumentation module. By using a time slot of $T_{0}=1998 \mu \mathrm{s}$, according to Equation (2), the PPM digital construction for a logic zero and logic one is computed as:

$$
m T_{0}=\frac{(-1)^{0}}{2}(1998)=999 \mu s m T_{0}=\frac{(-1)^{1}}{2}(1998)=-999 \mu s
$$

The construction of a logical one is defined by the position of the energy pulse to the first half of the time slot, $m T_{0}=-999 \mu \mathrm{s}$. Correspondingly, the second half of the time slot $m T_{0}=999 \mu$ s determines the logical zero. The microcontroller continuously generates 10-bit frames of guided waves PPM encoded energy pulses. The frame is comprised of a start pulse, eight pulses of data, and a stop pulse. The start and stop bits that delimitate each byte are made of 40 square pulses of $60 \mathrm{kHz}$, i.e., $666 \mu$ s. After the start bit, there is a set of 8 bits made of 20 square pulses of $60 \mathrm{kHz}$, i.e., $333 \mu$ s width each, carrying the actual digital information. Figure 8 illustrates this PPM symbol scheme.

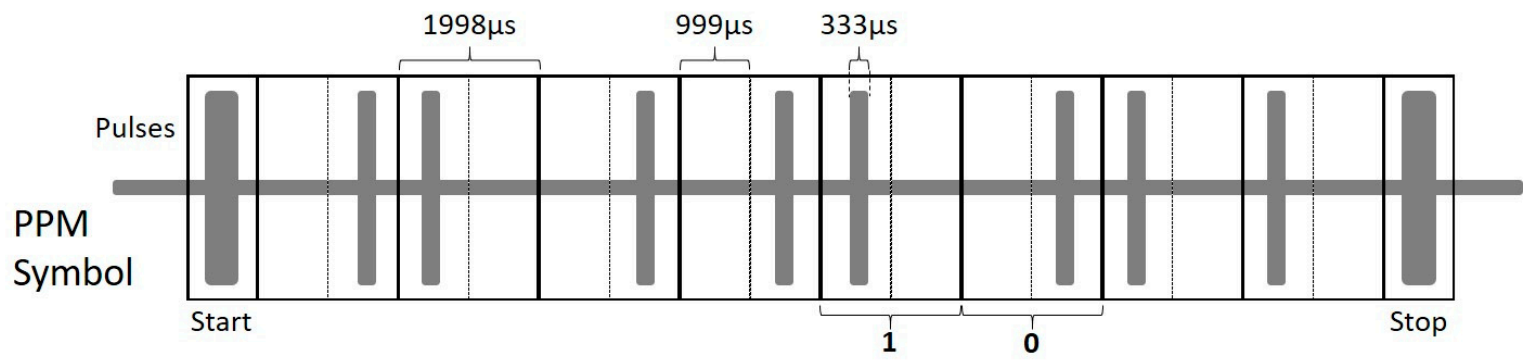

Figure 8. PPM symbol codification scheme for a sequence of 01001011 (4BH) data.

\subsection{PPM Signal Receiver}

The PPM guided wave receiver instrumentation package consists of a PZT-5H crystal, an instrumentation amplifier acting both as an amplifier and filter, a real-time LabVIEW based demodulator and data acquisition module, and an HMI used to set the system parameters and plot the results. Figure 9 depicts a block diagram of the PPM guided waves receiver instrumentation. A general description of the PPM receiver construction was recently carried out by [58].

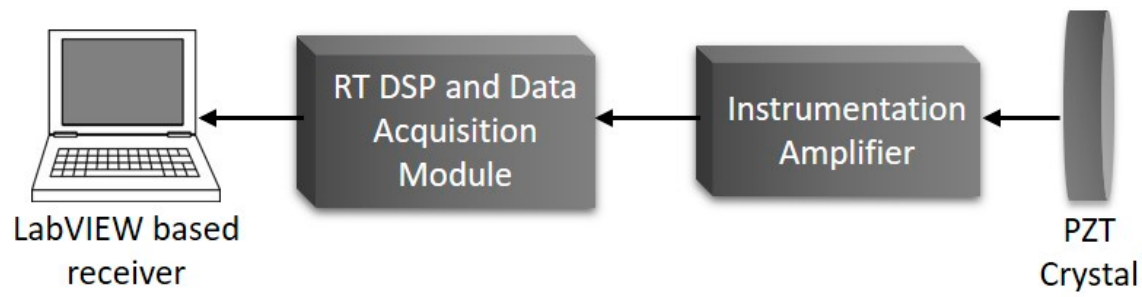

Figure 9. PPM guided waves receiver instrumentation block diagram.

One face of the PZT-5H crystal is connected to a low noise $(8 \eta \mathrm{V} / \sqrt{ } \mathrm{HZ})$ instrumentation amplifier that also performs as a low pass filter with a gain of ten and a cut-off frequency of $500 \mathrm{kHz}$ [59]. The instrumentation amplifier and the PZT-5H crystal are mounted in a cylindrical housing case such as the PPM transmitter. The other face of the PZT-5H disc is silicon glued to one side of a copper plate, and the other side is soldered to the other end of the central 1K22 MP-35N copper cables. The output of the instrumentation amplifier is connected to a commercial NI data acquisition module, which has a 16-bit analogue-to-digital converter with a $\pm 10 \mathrm{~V}$ analogue input and a sample rate up to $1 \mathrm{MHz}$. This 
module is interfaced to a real-time (RT) NI CompactRIO (cRIO) controller, which is used to acquire the PPM guided waves signals. The cRIO controller possesses a processor and programmable FPGA. This controller is optimized for programming code with LabVIEW that can be deployed onto both the RT operating system and directly onto the FPGA. The PPM demodulator and the digital signal processing algorithms run over the cRIO FPGA, which also is used to transmit the results and information to a personal computer via Ethernet to form the IHM.

\section{Digital Signal Processing, Signal Digitalization, and PPM Demodulation}

The PPM digitalization and demodulation processes are performed sequentially in three segments as depicted in the block diagram of Figure 10. First, the FPGA of the cRIO controller executes in real time a $1 \mathrm{MS} / \mathrm{s}$ data acquisition of the guided waves signals and carries out digital signal processing (DSP) operations such as a narrow band-pass filter and an autocorrelation-based function for noise suppressing and signal improvement. The blue signal depicted in Figure 10 represents the acquired signal coming from the instrumentation amplifier. The red curve stands for the output of the narrow band-pass filter, and the green signal embodies the autocorrelation of the filtered signal. In the second segment, the following two RT DSP operations are performed: a low pass finite impulse response (FIR) filter tuned to the transmission baud rate and a root mean square (RMS) threshold computation. The third segment of the process is accomplished offline to display information in the IHM. At the start, a comparison between the baud rate of the filtered signal and the RMS threshold value is carried out, subsequently, a post-processing PPM demodulation and checksum HASH byte validation are implemented. The magenta curve represents the output of the baud rate tuned filter and contains a horizontal crossing line that stands for the RMS threshold value. The cyan curve shows the digital signal in which every pulse represents the duration of each acoustic pulse, and the time between them conveys the PPM information. Finally, two arrays display in hexadecimal the actual PPM received data and their validation HASH byte.

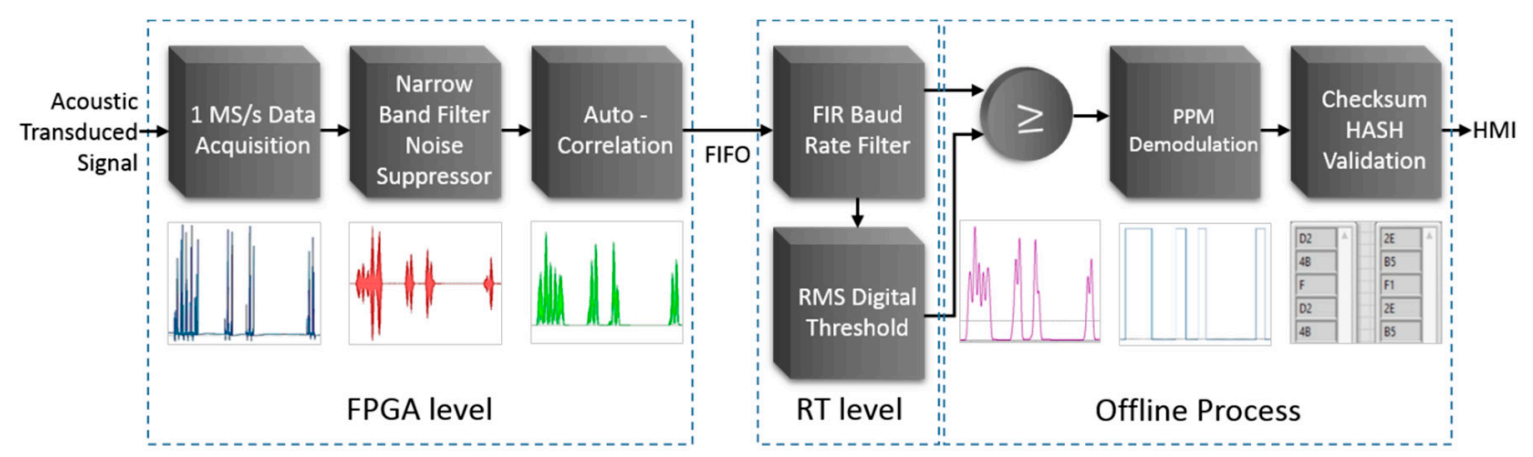

Figure 10. PPM digitalization and demodulation processes from data acquisition of the acoustic signals to byte validation.

The narrow band noise suppressor filter implemented in the RT FPGA performs a band-pass infinite impulse response (IIR) Butterworth filter, whose cut-off frequencies are 48 and $62 \mathrm{kHz}$. The general difference equation that characterizes an IIR filter is given by [60]

$$
y_{i}=\frac{1}{a_{0}}\left(\sum_{j=0}^{N_{b}-1} b_{j} x_{i-j}-\sum_{k=1}^{N_{a}-1} a_{k} y_{i-k}\right)
$$

where $b_{j}$ is the set of forward coefficients, $N_{b}$ is the number of forward coefficients, $a_{k}$ is the set of reverse coefficients, and $N_{a}$ is the number of reverse coefficients; and where $x_{i}$ is the current input, $x_{i-j}$ is the past inputs, and $y_{i-k}$ is the past outputs. 
Signal enhancement is attained by using an autocorrelation-based function, which can be expressed by [61]

$$
y(l)=\sum_{n=i}^{\langle N-|k|-1\rangle} x(n) x(n-l)
$$

where $i=l, k=0$ for $l \geq 0$, and $i=0, k=l$ for $l<0$. Index $l$ is a time shift or lag parameter. The disposition of this function presents two advantages: to stand out the guided waves pulses amplifying acoustic signal groups and to attenuate noise. The practical implementation of this operation is performed calculating the product of the received filtered signal $x(n)$ multiplied by itself delayed with a defined temporal displacement $x(n-l)$. The autocorrelation-based function multiplies positive slopes by themselves amplifying positive peaks. It also multiplies negative slopes by themselves that results in positive values amplified as positive peaks. As a result, the noise stays at a low amplitude level, while the acoustic pulses are amplified. This facilitates the identification of the acoustic pulses and provides well-defined amplified positive acoustic pulses ready to be filtered with a low-pass filter to unify them into a single pulse representing the width of a digital square pulse modulated in PPM.

The baud rate tuned filter implemented is the 129 taps FIR low-pass filter that is executed using the standard FIR convolution expression [61]

$$
y[n]=\sum_{k=0}^{N-1} h[k] x[n-k]
$$

where $y(n)$ and $x(n)$ correspond to the output and input signals, respectively, and the filter impulse response is denoted by $h(k)$. The coefficients of the filter are obtained using the frequency sampling method, whose algorithm is condensed by the expression

$$
h[n]=f_{w}[n] F^{-1}\{H[k]\}\left\{\begin{array}{c}
H_{r}[k]=1, f_{l}<k<f_{h} \\
H_{r}[k]=0, \text { elsewhere } \\
H_{i}[k]=0
\end{array}\right.
$$

where $f_{w}[n]$ denotes a window function and $H[k]$ characterizes the frequency response of the filter specified in the Fourier domain. The frequency response, for a linear phase filter, is determined by setting the real terms $\left(H_{r}[k]\right)$ to their intended values denoted by the low and high cut-off frequencies defined by the terms $f_{1}$ and $f_{h}$ and specifying the imaginary terms $H_{i}[k]$ as zero. The real-time digital filter was implemented with a bandwidth of $80 \mathrm{~Hz}$, whose cut off frequencies were $0 \mathrm{~Hz}$ and $80 \mathrm{~Hz}$, respectively, i.e., it was designed to operate to the actual transmission baud rate.

After the baud rate FIR filter is executed, a RMS function is computed using $30 \mathrm{k}$ samples for the digitalization process. The threshold value is characterized through the RMS value expressed by [62]

$$
V_{R M S}=\sqrt[2]{\frac{1}{N} \sum_{i=0}^{N-1}\left(x_{i}\right) 2}
$$

where the values $x_{i}$ are the samples of the periodic signal and exact $\mathrm{N}$ samples are equally spaced along one complete signal period.

Signal digitalization is attained by carrying out a comparison point-to-point between the baud rate low-pass filtered signal and the RMS threshold values. The resultant digital pulses accommodate the transmitted PPM encoded information. The demodulation processes commence by identifying the start and the stop pulses and quantifying the time delay between the previously digitalized pulses. Each bit depends on the digital value of the previous bit and the delay time between them. Discounting start and stops pulses, the demodulator identifies frames of 8 bits. The decoded bytes are stored in a two-dimensional array of 8 Booleans values per row that contain the actual transmitted bytes and their corresponding validation HASH bytes. The validation process separates the actual data bytes from the validation HASH bytes and performs the HASH calculation and validation. Demodulated data bytes 
are converted to hexadecimal bytes and stored in a one-dimensional (1D) array. Decoded HASH bytes are compared with their correspondent data bytes to identify transmission errors.

\section{Experimental Configuration and Automatic PPM Communication Results}

A series of experiments were conducted in the laboratory using two lengths of $1 \mathrm{~K} 22 \mathrm{MP}-35 \mathrm{~N}$ multi-conductor cables. The first set of experiments were aimed to identify the excited guided waves modes, $\mathrm{L}(0,1)$ and $\mathrm{F}(1,1)$, using a $1 \mathrm{~m}$ long cable. The second group of trials was conducted using 1 and $10 \mathrm{~m}$ long cables to automatically perform the transmission and reception of PPM symbols of 8-bit digital information. Each end of the $1 \mathrm{~K} 22 \mathrm{MP}-35 \mathrm{~N}$ inner copper multiwire cables, 18-AWG, was soldered to the 3 inch diameter copper plates mounted and fixed to the transmitter and the receiver instrumentation modules, respectively. The experimental setup for both experiments is illustrated in Figure 11.

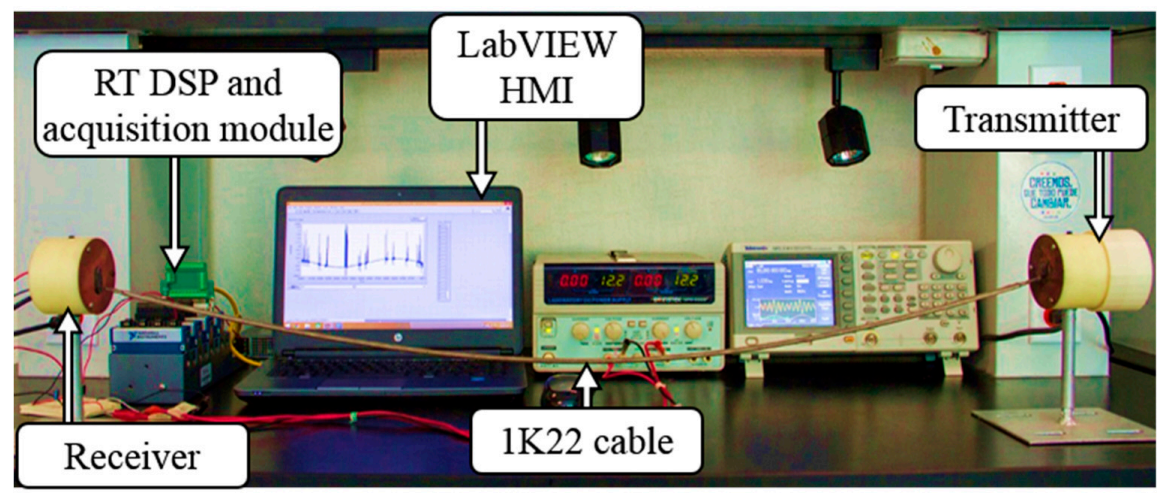

Figure 11. Experimental setup.

\subsection{Guided Wave Propagation Mode Identification}

In this experiment, the transmitter generated 20 square pulses of $60 \mathrm{kHz}$. A time domain analysis estimation of the time-of-arrival (TOA) of the received signal was implemented. The time difference between the transmitted and the received signal, over $1 \mathrm{~m}$ cable, gave an approximation of the propagation group velocity. Figure 12 illustrates both the transmitted square pulses (pale green curve) and the guided waves signals received signals (dark green curve).

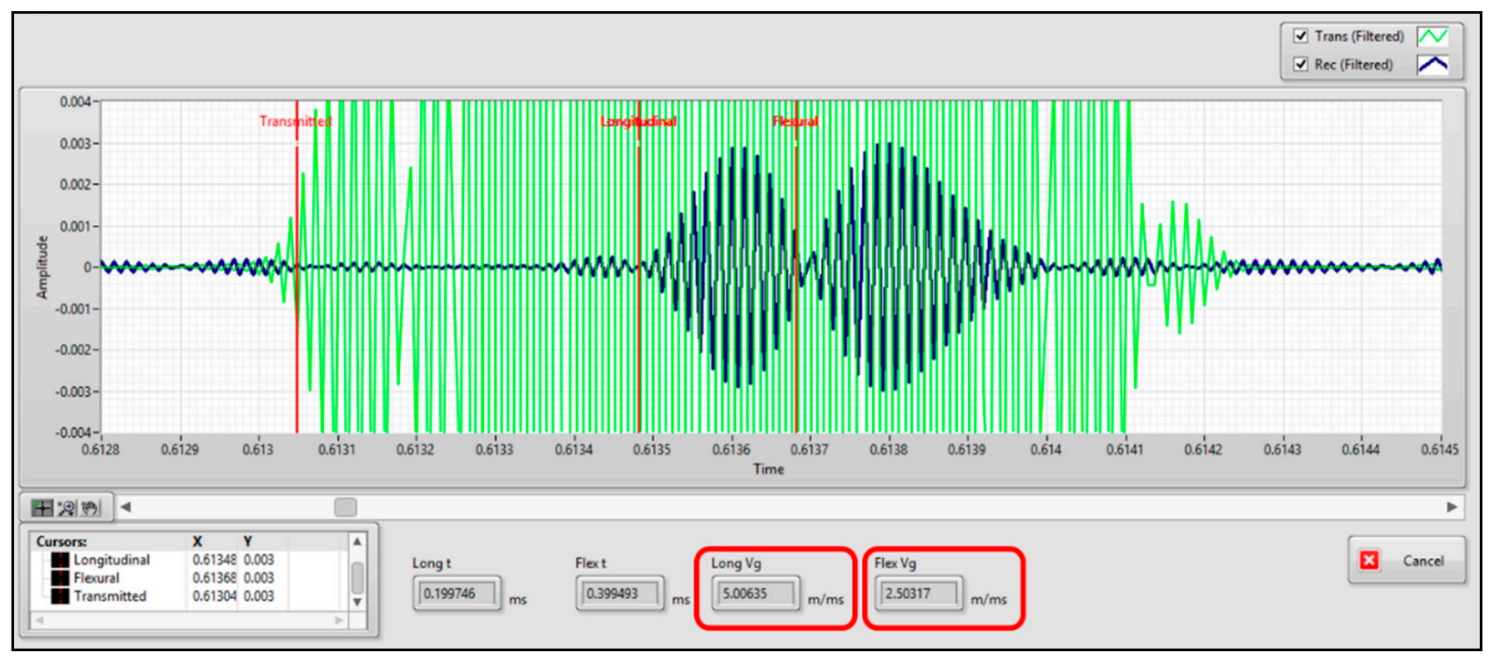

Figure 12. Guided wave propagation mode identification of a group of signals transmitted through $1 \mathrm{~m}$ of $1 \mathrm{~K} 22 \mathrm{MP}-35 \mathrm{~N}$ cable. The pale green curve shows the transmitted pulses and the dark green curve shows the excited guided waves modes. 
Two groups of signals with different group velocities were received. By using the LabVIEW HMI, it was observed that the first group of signals propagated at $5.00635 \mathrm{~m} / \mathrm{ms}$, and the second group propagated at $2.50317 \mathrm{~m} / \mathrm{ms}$. According to the dispersion curves in Figure $2 \mathrm{~b}$, two guided waves can be excited at $60 \mathrm{kHz}$, one at $4.999 \mathrm{~m} / \mathrm{ms}$, and the other at $2.435 \mathrm{~m} / \mathrm{ms}$. The received first group agrees to the longitudinal mode $\mathrm{L}(0,1)$, and the second group concurs to the flexural mode $\mathrm{F}(1,1)$; thereby it is possible to recognize the excited guided waves modes.

\subsection{Automatic Guided Waves PPM Communication Results}

In the second group of experiments, two lengths of multiwire cables were employed, 1 and 10 $\mathrm{m}$. The flash memory of the PPM instrumentation transmitter was programmed with three different symbols that were transmitted continuously once the instrumentation was powered. Digital signal processing and signal digitalization y PPM demodulation were performed automatically in the receiver instrumentation package. Figure 13 depicts the received DSP and signal digitalization process when the transmitter sends a symbol 4BH (01001011B).

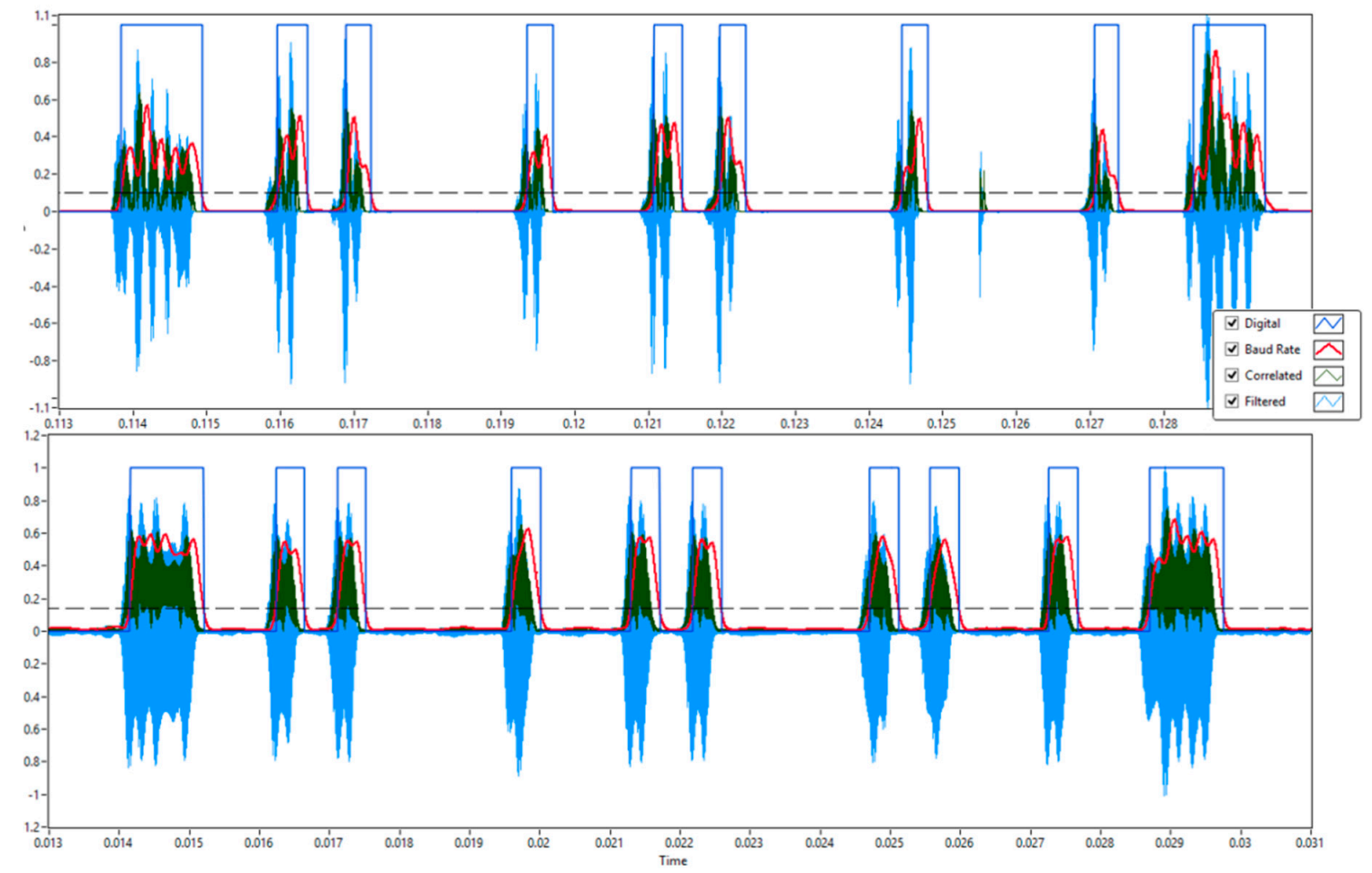

Figure 13. Digital signal processing (DSP) and signal digitalization of a symbol (4BH) identification over $1 \mathrm{~m}$ cable, upper trace, and $10 \mathrm{~m}$ cable, lower trace.

In both plots, cyan curves are the received acoustic guided waves signal band-pass filtered; green curves, are the correlated signal; red curves, are the baud rate filtered signal; the horizontal dotted black line is the RMS value; and the blue square pulses are the actual digital data encapsulated in an 8-bits symbol, including start and stop pulses. The attenuation between the 1 and $10 \mathrm{~m}$ transmissions is about $32 \mathrm{~dB}$; nevertheless, ultrasonic pulses are more uniform in the $10 \mathrm{~m}$ cable avoiding signal minimums under the threshold. In this case, the baud rate filter generates a smooth and thick positive peak (red curve) for each group of acoustic pulses. Although the energy loss is higher in the $10 \mathrm{~m}$ cable than in $1 \mathrm{~m}$ cable, the system provides sufficient SNR to successfully decode and demodulate the transmitted information.

The visualization of the intermediate DSP processes was omitted in the final tests to accelerate its processing at the FPGA level. Figure 14 shows six of groups of signals; in blue curves, the DSP acoustic signals, and in red curves, the digitalized acoustic pulses. These groups of signals correspond to the 
transmission of the three information bytes and their associated HASH bytes delayed $10 \mathrm{~ms}$. Each information byte and HASH byte are transmitted every $20 \mathrm{~ms}$.

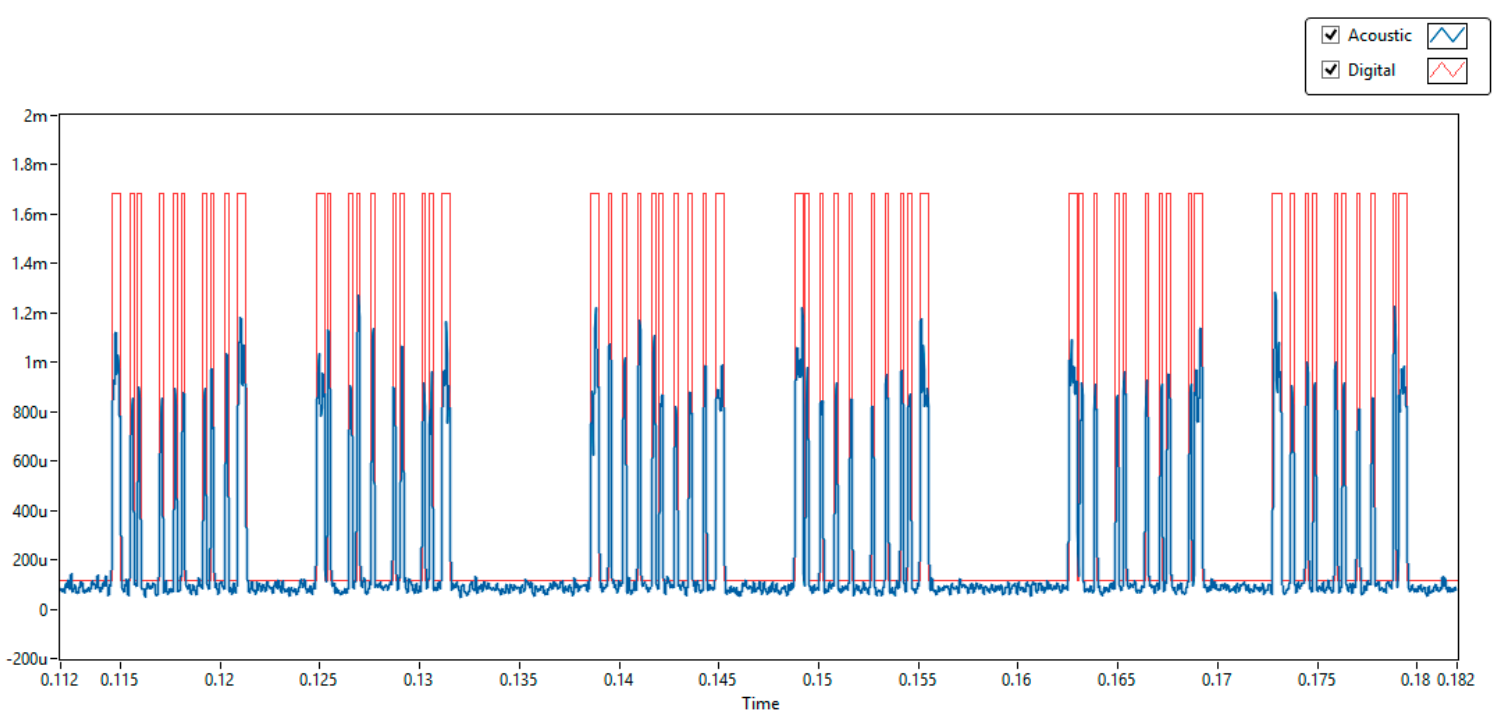

Figure 14. Identification of three different information bytes and their corresponding HASH bytes.

The automatic PPM demodulation results were visualized using the IHM developed in LabVIEW. Figure 15 depicts a two-dimensional (2D) array containing 8 bits per row, where the LSB (least significant bit) is located on the right side of the row. Every row represents the byte information that is continuously transmitted. The hexadecimal values of received bytes per row are stored in an array and displayed in the IHM.

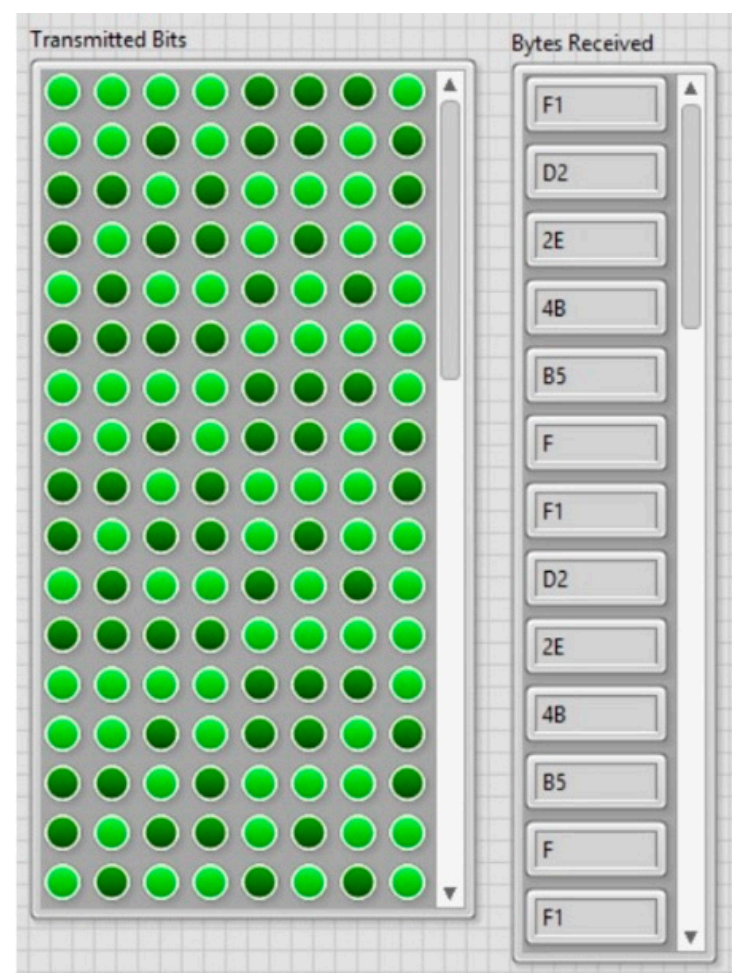

Figure 15. Two-dimensional (2D) array of 8 digital bits transmitted per row and its hexadecimal values of the received bytes. 
Figure 16 shows the transmitted information byte and its HASH byte, in two separated arrays. The HASH test validation results are shown in a separate string array. Each element of the array contains the string "Correct Byte" or "Transmission Error" depending on the equivalency between each data transmitted byte and its HASH byte.

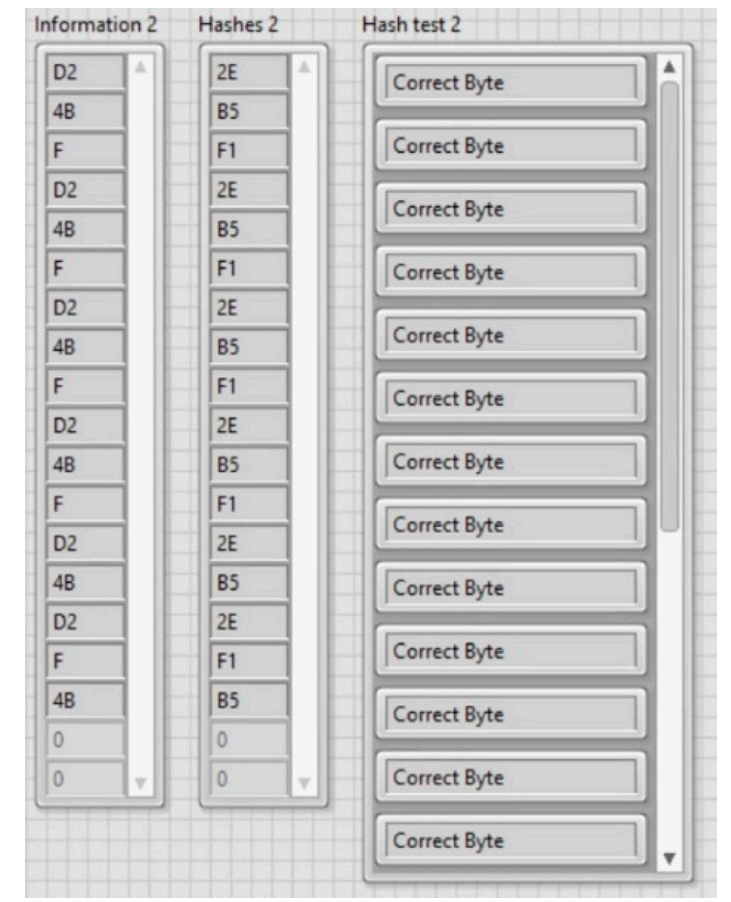

Figure 16. Received information bytes with its corresponding HASH bytes, and HASH validation.

As depicted in Figures 15 and 16, the automatic PPM demodulation process successfully identified, validated, and displayed the transmitted information in real time using 1 and $10 \mathrm{~m}$ 1K22 MP-35N multiwire cables as a communication channel. Data transmission rates, up to 470 bps, were experimentally obtained, which correspond to the selected acoustic propagation frequency of 60 $\mathrm{kHz}$. The average byte error rate of the communication system was around $5 \%$ for $10 \mathrm{~m}$ transmission under laboratory conditions.

\section{Discussion and Further Work}

An automatic ultrasonic guided waves PPM data transmission system using high-temperature, high-pressure, and corrosion-resistant $1 \mathrm{~K} 22 \mathrm{MP}-35 \mathrm{~N}$ multiwire cables as a communication channel has been designed, implemented, and evaluated. The system uses cables commonly employed in the hydrocarbon industry for downhole data acquisition from producing wells, which are often immersed in harsh environments. The complicated mechanical structures of the $1 \mathrm{~K} 22 \mathrm{MP}-35 \mathrm{~N}$ multiwire cable was modeled as concentric rod made of copper, an isolation layer and steel. Dispersion curves of this concentric rod were calculated using the software Disperse. The identification of guided waves modes, $\mathrm{L}(0,1)$ and $\mathrm{F}(1,1)$, at $60 \mathrm{kHz}$ and over a $1 \mathrm{~m}$ multiwire cable, were validated in the laboratory.

A battery-powered stand-alone instrumentation PPM transmitter made of a microcontroller, a signal booster, and a piezo ceramic PZT-5H disc was implemented. Three different byte-information symbols with frames of 10 bits were programmed in the internal flash memory of the microcontroller and were transmitted continuously once the instrumentation was powered. The receiver instrumentation package consisted of a PZT-5H disc, an instrumentation amplifier, and a 16-bit and $1 \mathrm{MS} / \mathrm{s}$ data acquisition module interfaced to an RT cRIO controller. The cRIO controller not only executed DSP algorithms, signal digitalization, and the required PPM demodulation process but also transmitted the results and information to a personal computer via Ethernet to an IHM for displaying the attained 
results. A proprietary communication protocol at physical and data link layers, including real-time DSP filtering, PPM encoding, PPM decoding, and HASH byte validation was presented.

Experiments were carried out under laboratory conditions using 1 and $10 \mathrm{~m} 1 \mathrm{~K} 22 \mathrm{MP}-35 \mathrm{~N}$ multiwire cables. Results show that by exploring the waveguide effect of multi-conductor cables acting as a communication channel, successful transmission, reception, and validation of $60 \mathrm{kHz}$ encoded PPM digital information has been achieved. The communication system and protocol show data transmission rates up to 470 bps with an average byte error rate around 5\%. Although the trials have been conducted over small distances, the acoustic guided waves digital data transmission has been demonstrated by the feasibility of detecting and decoding in real-time dispersive guided waves energy packets, provided sufficient SNR. The implemented communication protocol suggests that a non-complex modulation, such as PPM, is sufficient to transmit digital information. Although data transmissions present occasional loss bits, increasing the robustness of the communication protocol recommends a better performance for the digital communication, for example, using bit codification techniques, redundancy, and adaptive modulations, which is proposed as future work. These results form the basis for future development of a guided waves data transmission system for automatic monitoring purposes using longer mono-conductor multiwire cables immersed in oil and water to resemble bottom hole conditions.

Author Contributions: Conceptualization, R.M.; data curation, G.T.; investigation, G.T.; methodology, R.M.; resources, J.A.P.-D.; software, G.T.; supervision, R.M.; validation, G.T. and R.M.; visualization, J.A.P.-D.; writing-original draft, G.T.; writing - review and editing, R.M. and J.A.P.-D. All authors have read and agreed to the published version of the manuscript.

Funding: This research received no external funding.

Conflicts of Interest: The authors declare no conflict of interest.

\section{References}

1. Serra, O. Fundamentals of Well-Log Interpretation, The Acquisition of Logging Data; Elsevier Science Publishers B. V.: Amsterdam, The Netherlands, 1984.

2. Ramesh, A. Well Logging Principles, Interpretation and Applications. Master's Thesis, Dr. B.R. Ambedkar University, Srikakulam, India, 2016.

3. Liu, Y. Interpreting Pressure and Flow Rate Data from Permanent Downhole Gauges Using Data Mining Approaches. Ph.D. Thesis, University of Stanford, Stanford, CA, USA, 2013. Available online: http: //purl.stanford.edu/xp635wx9603 (accessed on 4 February 2020).

4. Petroleum and Natural Gas Industries-Fixed Steel Offshore Structures. ISO 19902:2007; Geneva, Switzerland, December 2007. Available online: https://www.iso.org/standard/27507.html (accessed on 4 February 2020).

5. Amani, M.; Al-Jubouri, M.; Shadravan, A. Comparative Study of Using Oil-Based Mud Versus Water-Based Mud in HPHT Fields. Adv. Pet. Explor. Dev. 2012, 4, 18-27. [CrossRef]

6. Belaigues, A.; Paumard, A.; Durand, Y.; Calvert, T.J. Well Logging Communication System. U.S. Patent 4,355,310, 19 October 1982.

7. Mijarez, R.; Pascacio, D.; Guevara, R.; Rodriguez, J. Signal processing algorithm for thermal drift compensation in high temperature down-hole instrumentation systems. Trans. Inst. Meas. Control 2017, 39, 1161-1168. [CrossRef]

8. Mijarez, R.; Pascacio, D.; Guevara, R.; Rodríguez, J.H.; Pacheco, M.J.O. Downhole Intelligent Communication System Based on Real Time Characterization of Signal Attenuation in Coaxial Cable Used as Transmission Medium. U.S. Patent US9537606B2, 3 January 2017.

9. Kessler, S.S. Certifying a structural health monitoring system: Characterising durability, reliability and longevity. In Proceedings of the 1st International Forum on Integrated Systems Health Engineering and Management in Aerospace, Napa, CA, USA, 7-10 November 2005; pp. 1-7.

10. Cawley, P. Structural health monitoring: Closing the gap between research and industrial deployment. Struct. Health Monit. 2018, 17, 1225-1244. [CrossRef]

11. Mitra, M.; Gopalakrishnan, S. Guided wave based structural health monitoring: A review. Smart Mater. Struct. 2016, 25, 053001. [CrossRef] 
12. Rizzo, P. Ultrasonic Wave Propagation in Progressively Loaded Multi-Wire Strands. Exp. Mech. 2006, 46, 297-306. [CrossRef]

13. Baltazar, A.; Hernandez-Salazar, C.D.; Manzanares-Martinez, B. Study of wave propagation in a multiwire cable to determine structural damage. NDT E Int. 2010, 43, 726-732. [CrossRef]

14. Mijarez, R.; Baltazar, A.; Rodríguez Rodríguez, J.; Ramírez-Niño, J. Damage detection in ACSR cables based on ultrasonic guided waves. Dyna 2014, 81, 226-233. [CrossRef]

15. Ibáñez, F.; Baltazar, A.; Mijarez, R. Detection of Damage in Multiwire Cables Based On Wavelet Entropy Evolution. Smart Mater. Struct. 2015, 24, 085036. [CrossRef]

16. Legg, M.; Yücel, M.K.; Kappatos, V.; Selcuk, C.; Gan, T.-H. Increased range of ultrasonic guided wave testing of overhead transmission line cables using dispersion compensation. Ultrasonics 2015, 62, 35-45. [CrossRef] [PubMed]

17. Schaal, C.; Bischoff, S.; Gaul, L. Damage Detection in Multi-Wire Cables Using Guided Ultrasonic Waves. Struct. Health Monit. 2016, 15, 279-288. [CrossRef]

18. Cheng, J.; Qiu, J.; Ji, H.; Wang, E.; Takagi, T.; Uchimoto, T. High Precision Ultrasonic Guided Wave Technique for Inspection of Power Transmission Line. Chin. J. Mech. Eng. 2017, 30, 170-179. [CrossRef]

19. Zhang, P.; Tang, Z.; Lv, F.; Yang, K. Numerical and Experimental Investigation of Guided Wave Propagation in a Multi-Wire Cable. Appl. Sci. 2019, 9, 1028. [CrossRef]

20. Drumheller, D.S.; Kuszmaul, S.S. Acoustic Telemetry; Technical Report SAND2003-2614; Sandia National Laboratories: Albuquerque, NM, USA, 2003. [CrossRef]

21. Mijarez, R.; Gaydecki, P.; Burdekin, M. Continuous monitoring guided wave encoded sensor for oil rig flooded member detection. Insight Non-Destruct. Test. Cond. Monit. 2005, 47, 748-751. [CrossRef]

22. Memarzadeh, M. Optimal Bore-Hole Communication Using Multi-Carrier Modulation. Ph.D. Thesis, Rice University, Houston, TX, USA, 2007. Available online: https://scholarship.rice.edu/handle/1911/20631 (accessed on 4 February 2020).

23. Mijarez, R.; Gaydecki, P. Automatic guided wave PPM communication system for potential SHM of flooding members in sub-sea oil rigs. Smart Mater. Struct. 2013, 22, 055031. [CrossRef]

24. Jin, Y.; Ying, Y.; Zhao, D. Data communications using guided elastic waves by time reversal pulse position modulation: Experimental study. Sensors 2013, 13, 8352-8376. [CrossRef] [PubMed]

25. Chakraborty, S.; Saulnier, G.J.; Wilt, K.W.; Curt, E.; Scarton, H.A.; Litman, R.B. Low-power, low-rate ultrasonic communications system transmitting axially along a cylindrical pipe using transverse waves. IEEE Trans. Ultrason. Ferroelectr. Freq. Control 2015, 62, 1788-1796. [CrossRef] [PubMed]

26. Huang, X.; Saniie, J.; Bakhtiari, S.; Heifetz, A. Applying EMAT for ultrasonic communication through steel plates and pipes. In Proceedings of the IEEE International Conference on Electro/Information Technology, Rochester, MI, USA, 3-5 May 2018; pp. 0379-0383.

27. Wu, A.; He, S.; Ren, Y.; Wang, N.; Ho, S.C.H.; Song, G. Design of a New Stress Wave-Based Pulse Position Modulation (PPM) Communication System with Piezoceramic Transducers. Sensors 2019, 19, 558. [CrossRef]

28. Shoudy, D.; Saulnier, G.J.; Scarton, H.A.; Das, P.K.; Roa-Prada, S.; Ashdown, J.D.; Gavens, A.J. An Ultrasonic Through-Wall Communication System with Power Harvesting. In Proceedings of the IEEE Ultrasonics, Symposium Proceedings, New York City, NY, USA, 28-31 October 2007; pp. 1848-1853.

29. Primerano, R.; Kam, M.; Dandekar, K. High bit rate ultrasonic communication through metal channels. In Proceedings of the IEEE 43rd Annual Conference on Information Sciences and Systems, Baltimore, MD, USA, 18-20 March 2009; pp. 902-906.

30. Hosman, T.; Yeary, M.; Antonio, J.K. Design and characterisation of an MFSK-based transmitter/receiver for ultrasonic communication through metallic structures. IEEE Trans. Instrum. Meas. 2011, 60, 3767-3774. [CrossRef]

31. Ashdown, J.D.; Saulnier, G.J.; Lawry, T.J.; Wilt, K.R.; Scarton, H.A.; Pascarelle, S.; Pinezich, J.D. Multi-Channel Data Communication Through Thick Metallic Barriers. In Proceedings of the IEEE International Conference on Communications, Ottawa, ON, Canada, 10-15 June 2012; pp. 4678-4683.

32. Lawry, T.J.; Saulnier, G.J.; Ashdown, J.D.; Scarton, H.A.; Gavens, A. A Full-Duplex Ultrasonic Through-Wall Communication and Power Delivery System with Frequency Tracking. U.S. Patent US9455791, 27 September 2016.

33. Yang, D.; Hu, Z.; Zhao, H.; Hu, H.; Sun, Y.; Hou, B. Through-Metal-Wall Power Delivery and Data Transmission for Enclosed Sensors: A Review. Sensors 2015, 15, 31581-31605. [CrossRef] [PubMed] 
34. Primerano, R.A.; Kam, M.; Dandekar, K.R.; Gindhart, C. System for Ultrasonic Communication Across Curved Metal Surfaces. U.S. Patent US0163354 A1, 8 June 2017.

35. Ashdown, J.D.; Liu, L.; Saulnier, G.J.; Wilt, K.R. High-Rate Ultrasonic Through-Wall Communications Using MIMO-OFDM. IEEE Trans. Commun. 2018, 66, 3381-3393. [CrossRef]

36. Zhang, J.; Yu, Z.; Yang, H.; Wu, M.; Yang, J. Ultrasonic Wireless Communication Through Metal Barriers. Sound Vib. 2019, 53, 2-15. [CrossRef]

37. Moll, J.; De Marchi, L.; Marzani, A. Transducer-to-Transducer Communication in Guided Wave Based Structural Health Monitoring. In Proceedings of the 19th World Conference on Non-Destructive Testing, Munich, Germany, 13-17 June 2016; pp. 1-8.

38. Moll, J.; Mälzer, M.; De Marchi, L.; Testoni, N.; Marzani, A. Experimental Analysis of Digital Data Communication in Intelligent Structures Using Lamb Waves. In Proceedings of the 11th International Workshop on Structural Health Monitoring, Stanford, CA, USA, 12-14 September 2017; pp. 1654-1661.

39. Moll, J.; Kexel, C.; Mälzer, M. Complex Intelligent Structures with Data Communication Capabilities. In Proceedings of the 9th European Workshop on Structural Health Monitoring, Manchester, UK, 10-13 July 2018; pp. 1-7.

40. Kexel, C.; Mälzer, M.; Moll, J. Guided Wave Based Acoustic Communications in Structural Health Monitoring Systems in the Presence of Structural Defects. In Proceedings of the IEEE International Symposium on Circuits and Systems, Florence, Italy, 27-30 May 2018; pp. 1-4.

41. Wang, B.; Saniie, J.; Bakhtiari, S.; Heifetz, A. Software Defined Ultrasonic System for Communication Through Solid Structures. In Proceedings of the IEEE International Conference on Electro/Information Technology, Rochester, MI, USA, 3-5 May 2018; pp. 1-4.

42. Kexel, C.; Kexel, C.; Maetz, T.; Mälzer, M.; Moll, J. Digital communication across orthotropic composite components using guided waves. Compos. Struct. 2019, 209, 481-489. [CrossRef]

43. Mateo, C.; Talavera, J.A.; Muñoz, A. Elastic Guided Wave Propagation in Electrical Cables. IEEE Trans. Ultrason. Ferroelectr. Freq. Control 2007, 54, 1423-1429. [CrossRef]

44. Mateo, C. Modos de Propagación de las Ondas Elásticas en los Cables Eléctricos. Ph.D. Thesis, Universidad Pontificia Comillas de Madrid, Madrid, Spain, 2007. (In Spanish).

45. Trane, G.; Mijarez, R.; Guevara, R.; Baltazar, A. PZT guided waves sensor permanently attached on multi-wire AWG12 cables used as communication medium. AIP Conf. Proc. 2015, 1650, 631-639.

46. Trane, G.; Mijarez, R. Guided waves PPM encoded system using AWG12 cables as communication channel. In Proceedings of the 11th European Conference on Non-Destructive Testing (ECNDT 2014), Prague, Czech Republic, 6-10 October 2014; pp. 1-8.

47. Trane, G.; Mijarez, R.; Guevara, R.; Pascacio, D. PPM-based system for guided waves communication through corrosion resistant multi-wire cables. Phys. Procedia 2015, 70, 672-675. [CrossRef]

48. CAMESA EMC Product Catalogue-7/32" (5.69 mm) Monoconductor 1K22. Available online: http: //www.camesaemc.com/Resource_/PageResource/Camesa/1K22\%20MP35N.pdf (accessed on 5 February 2020).

49. Graff, K.F. Wave Motion in Elastic Solids; Dover Pbs.: New York, NY, USA, 1991.

50. Pavlakovic, B.; Lowe, M. Disperse User Manual: A System for Generating Dispersion Curves; Imperial College, University of London: London, UK, 2003.

51. Cawley, P.; Lowe, M.; Alleyne, D.N.; Pavlakovic, B.; Wilcox, P. Practical long-range guided wave testing: Applications to pipes and rail. Mater. Eval. 2003, 61, 66-74.

52. Cawley, P.; Alleyne, D. The use of Lamb waves for the long-range inspection of large structures. Ultrasonics 1996, 34, 287-290. [CrossRef]

53. Pollakowski, M.; Ermert, H. Chirp signal matching and signal power optimisation in pulse-echo mode ultrasonic non-destructive testing. IEEE Trans. Ultrason. Ferroelectr. Freq. Control 1994, 41, 655-659. [CrossRef]

54. McAulay, R.J. Optimal control techniques applied to PPM signal design. Inf. Control 1968, 12, $221-235$. [CrossRef]

55. Hamkins, J.; Moision, B. Selection of modulation and codes for deep-space optical communications. In Proceedings of the of SPIE, Free-Space Laser Communication Technologies XVI, San Jose, CA, USA, 16 June 2004; pp. 123-130.

56. Wozencraft, J.M.; Jacobs, I.M. Principles of Communication Engineering; Waveland Press, Inc.: Long Grove, IL, USA, 1990. 
57. Trane, G.; Mijarez, R.; Guevara, R.; Baltazar, A. Guided wave sensor for simple digital communication through an oil industry multi-wire cable. Insight Non-Destruct. Test. Cond. Monit. 2018, 60, $206-211$. [CrossRef]

58. Trane, G.; Mijarez, R.; Guevara, R.; Perez, A. A simplex guided wave communication system using oil industry multi-wire cable. In Proceedings of the 9th European Workshop on Structural Health Monitoring, Manchester, UK, 10-13 July 2018; pp. 1-12.

59. Mijarez, R. Electronica; Grupo Editorial Patria: México, D.F., Mexico, 2014. (In Spanish)

60. Singh, Y.; Tripathi, S.; Pandey, M. Analysis of digital IIR filter with Labview. Int. J. Comput. Appl. 2010, 10, 23-30. [CrossRef]

61. Gaydecki, P. Foundations of Digital Signal Processing: Theory, Algorithms and Hardware Design; The Institute of Electrical Engineers: London, UK, 2004.

62. Albu, M.; Heydt, G.T. On the rms values in power quality assessment. IEEE Trans. Power Deliv. 2003, 18, 1586-1587. [CrossRef]

(C) 2020 by the authors. Licensee MDPI, Basel, Switzerland. This article is an open access article distributed under the terms and conditions of the Creative Commons Attribution (CC BY) license (http://creativecommons.org/licenses/by/4.0/). 